\title{
PFCS: Pre-buffering-aware Flow Control Scheme for LTE-Advanced Relay Networks
}

\author{
Ping-Chen Lin*, Ray-Guang Cheng*, Xian Wang ${ }^{\dagger}$, Putu Ayu Rhamani Suryadhi* \\ ${ }^{*}$ Department of Electronic and Computer Engineering \\ National Taiwan University of Science and Technology, Taipei, Taiwan, R.O.C. \\ ${ }^{\dagger}$ Institute of Mobile Communications, Southwest Jiaotong University, China
}

\begin{abstract}
Relay technology is one of the techniques proposed to enhance the capacity of Long Term Evolution-Advanced (LTEA) networks. In an LTE-A relay network, each serving relay node has to store packets for its mobile users and forward the remaining packets to another node during handover. However, increasing the number of forwarded packets will result in the degradation of the users throughput. Flow control and prebuffering schemes are the two main schemes proposed to reduce the number of forwarded packets in LTE-A relay networks. Existing studies assumes the two schemes are independently operated. However, ignoring the effect of buffer overflow at the pre-buffering queue in a flow control scheme may result in ineffective operation. This paper presents a pre-bufferingaware flow control scheme (PFCS) for an LTE-A relay network that jointly considers the effects of both flow control and prebuffering schemes. The proposed PFCS can significantly improve user's throughput by preventing both buffer overflow and buffer underflow of the local queue at the serving relay node and minimizing the number of forwarded packets. Simulation results show that the PFCS can provide a better throughput than the traditional flow control schemes because the effect of the prebuffering scheme can minimize the number of forwarded packets.
\end{abstract}

Index Terms-LTE-Advanced, Relay, Pre-buffering scheme, Flow control scheme

\section{INTRODUCTION}

Relay technology is a candidate solution proposed by the 3rd Generation Partnership Project (3GPP) to extend the coverage or enhance the throughput for Long Term EvolutionAdvanced (LTE-A) networks. In LTE-A relay networks, DeNB does not know the UEs channel quality information (CQI) and each $\mathrm{RN}$ is responsible for relaying packets from the base station (called the donor eNode-B, DeNB) to its user equipment (UE). This results in a mismatch in the data rates between the relay link (wireless link connecting a DeNB and an RN) and the access link (wireless link between a DeNB and a UE or between an RN and a UE), and leads to problems of buffer overflow and buffer underflow at the RN. As a result, some UEs may experience excess packet loss due to buffer overflow, whereas some UEs may suffer from unexpected service interruption due to buffer underflow [4].

The 3GPP standard defines two types of RNs in LTE-A networks [1], [2]. A Type-I RN has its own cell identity (CID) and acts as a base station. It transmits its own common reference signal, broadcast information and scheduling information. A Type-II RN does not have its own CID and acts as a repeater. With a RN of a Type-I RN, the UE in an LTE-A relay network may handover to another RN. The handover from a serving RN should forward the buffered packets through a wireless link to the target cell. Increasing the number of forwarded packets may waste more uplink bandwidth [5]. This results in the degradation of network performance, which can be referred to as the handover forwarding problem.

A number of studies proposed some relative solutions to cope with these problems. Lin et al. [4] utilized a small buffer for each UE to relieve the handover forwarding problem. They proposed a dynamic flow control algorithm (DFCA) by dynamically adjusting the window size of flow control and the feedback frequency based on the measured signal quality of each UE. Lin et al. [8] studied the buffer overflow issue in HSDPA. They proposed the overflow control mechanisms via multicasting, since HSDPA allows a radio network controller (RNC) to pre-buffer the data packets for active cells. Kim and Cho [9] proposed a threshold-based scheme, called the pre-buffering scheme (PBS), in relay-based cellular systems. This scheme allows a base station to perform the multicast transmission to the candidate $\mathrm{RN}(\mathrm{s})$ when the received signal quality of the candidate $\mathrm{RN}$ is higher than a pre-defined threshold for the PBS.

Lin and Cheng [5] proposed flow control scheme, named the dynamic two-threshold flow control scheme (DTFCS), to deal with the buffer overflow and handover forwarding problems by dynamically adjusting the upper buffer threshold based on the users channel quality. However, the authors in [5] ignored the existence of uplink data traffic, and the forwarded packets can utilize only the remaining bandwidth of uplink transmission. Badache and Tandjaoui [6] proposed a new handoff protocol via bicasting to achieve a seamless handoff performance for mobile Internet Protocol version 4 (IPv4). Tanno et al. [7] also proposed a handover scheme that combines the method of bicasting and forwarding to reduce the handover delay and the amount of IP packet traffic.

This paper presents a pre-buffering-aware flow control scheme (PFCS) that controls the flow between the DeNB and RNs by considering the pre-buffering effect, to improve the users throughput in LTE-A relay networks. This paper is organized as follows. In Section 2, we present the system model of the LTE-A relay network considered in this paper. Section 3 describes the detailed design for the proposed scheme. Section 4 shows the simulation results to verify the effectiveness of the proposed scheme. Finally, Section 5 concludes the paper. 


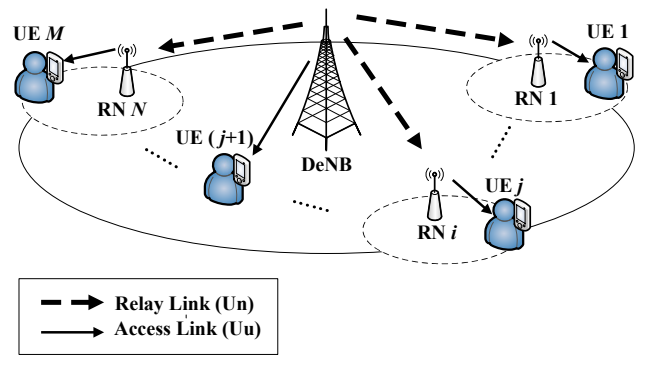

Fig. 1. System model for LTE-A relay networks.

\section{SySTEM MODEL}

Figure 1 shows an LTE-A relay network consisting of $N$ fixed RNs, $M$ mobile UEs, and one fixed DeNB. In such a network, a UE $j(1 \leq j \leq M)$ can either be served by a DeNB directly or served by an $\mathrm{RN} i$ ( where $1 \leq i \leq N$ ) passing through two independent radio links.

This study uses some basic assumptions. Each serving cell (e.g., DeNB or RN) allocates a finite local queue with length $K_{i, j}$ to UE $j$. For the pre-buffering functionality of the proposed scheme, each cell is further allocated a pre-buffering queue with length $L_{i}$ shared by all UEs served by RN $i$. Each UE can perform the handover when the measured signal-tonoise ratio (SNR) of a UE $j$ served by $\mathrm{RN} i, \gamma_{i, j}[k]$ is lower than a handover threshold $\gamma_{H O}$. An adaptive modulation and coding (AMC) scheme is used in each cell to maximize the system throughput. Table I [5] gives the detailed configuration of the LTE-A relay network. In downlink transmission, all source traffic of UE $j$ is generated from wired backhaul with rate $\lambda_{j}$ and is forwarded to the serving cell through a DeNB.

In an LTE-A relay network, we assume that a finite local queue with the maximum length $K_{i, j}$ is allocated in RN $i$ for UE $j$. We consider two transmission phases: the transmission of the relay link and the transmission of the access link. During the first phase, $A_{i, j}[k]$ packets are transmitted from the DeNB to $\mathrm{RN} i$ and buffered at the local queue of $\mathrm{RN} i$, where there are $B_{i, j}[k-1]$ packets already stored at the local queue of RN $i$ for UE $j$. During the second phase, $D_{i, j}[k]$ packets are transmitted from RN $i$ to UE $j$ and are served by RN $i$. Hence, the current buffer status of the local queue, $B_{i, j}[k]$, depends on the input $A_{i, j}[k]$, the output $D_{i, j}[k]$, the last buffer status of local queue $B_{i, j}[k-1]$, and the maximum length of the local queue $K_{i, j}$. Hence, we can estimate the current buffer

TABLE I

MOdulation AND CODING SCHEMES FOR LTE-A [5]

\begin{tabular}{cccc}
\hline \hline level & MCS & $R_{n}$ (bits/symbol) & $\gamma_{n}(\mathrm{~dB})$ \\
\hline 0 & Silent & 0 & 0 \\
1 & QPSK $(1 / 2)$ & 1 & 6 \\
2 & 16QAM $(1 / 2)$ & 2 & 11.5 \\
3 & 16QAM $(3 / 4)$ & 3 & 15 \\
4 & 64QAM $(2 / 3)$ & 4 & 18.5 \\
5 & 64QAM $(5 / 6)$ & 5 & 23 \\
\hline \hline
\end{tabular}

status of the local queue at RN $i$ for UE $j$ at the $k$ th subframe as

$$
\begin{array}{r}
B_{i, j}[k]=\max \left\{0, \min \left\{K_{i, j}, B_{i, j}[k-1]\right.\right. \\
\left.\left.+A_{i, j}[k]-D_{i, j}[k]\right\}\right\} .
\end{array}
$$

We find that when the channel quality of the access link is poorer than that of the relay link, $B_{i, j}[k]$ can easily reach $K_{i, j}$ and buffer overflow may occur. On the other hand, the buffer status $B_{i, j}[k]$ may reach zero and buffer underflow may emerge when the access link has a better channel quality than the relay link.

Based on (1), we can evaluate the probabilities of both buffer overflow and buffer underflow. To evaluate these probabilities, the capacity for UE $j$ dropped and unserved at the $k$ th subframe (unit: packet) is derived as

$$
\begin{aligned}
B_{i, j}^{(O)}[k]= & \max \left\{0, B_{i, j}[k-1]+A_{i, j}[k]\right. \\
& \left.-D_{i, j}[k]-K_{i, j}\right\}, \\
B_{i, j}^{(U)}[k]= & \max \left\{0, D_{i, j}[k]-B_{i, j}[k-1]\right. \\
& \left.-A_{i, j}[k]\right\} .
\end{aligned}
$$

The buffer overflow probability $P^{(O)}$ can be derived from the sum of the number of dropped packets, $B_{i, j}^{(O)}[k]$, over the total number of arrival packets from the DeNB, which is given by

$$
P^{(O)}=\frac{\frac{1}{T} \sum_{k=1}^{T} \sum_{i=1}^{N} \sum_{j=1}^{M} B_{i, j}^{(O)}[k]}{\frac{1}{T} \sum_{k=1}^{T} \sum_{j=1}^{M} \lambda_{j}[k]},
$$

where $T$ and $\lambda_{j}[k]$ are an observation interval and the number of arrival packets for UE $j$ at the $k$ th subframe, respectively.

The buffer underflow probability $P^{(U)}$ can be derived from the sum of the number of unserved packets, $B_{i, j}^{(U)}[k]$, over the total service capacity in the access link, which is given by

$$
P^{(U)}=\frac{\frac{1}{T} \sum_{k=1}^{T} \sum_{i=1}^{N} \sum_{j=1}^{M} B_{i, j}^{(U)}[k]}{\frac{1}{T} \sum_{k=1}^{T} \sum_{i=1}^{N} \sum_{j=1}^{M} D_{i, j}[k]} .
$$

Due to the handover in LTE-A relay networks, the number of forwarded packets, $B_{\tau(j), j}[k]$, is another key performance metric to evaluate the efficiency of a flow control scheme. Let $H_{j}$ be sets of subframes that UE $j$ hands over from RN $i$ to the new target cell $\tau(j)$. All packets, for a single UE $j$, buffered at RN $i$ should be forwarded. Hence, we can estimate the average number of forwarded packets in an LTE-A relay network, $F$, as

$$
F=\frac{1}{T} \sum_{j=1}^{M} \sum_{k \in H_{j}} B_{\tau(j), j}[k] .
$$


Because of user's mobility, an UE may perform handover and can be served by different cells. There are five transmission cases in an LTE-A relay network:

Case 1All packets are received from the serving DeNB.

Case 2All packets are received from the serving RN.

Case 3All forwarded packets are received when the handover from the RN to the DeNB is performed.

Case 4All forwarded packets are received when the handover between two RNs is performed.

Case 5All forwarded packets are received when the handover from the DeNB to the RN is performed.

It is worth noting that transmission cases 2 and 5 are exactly the same. Hence, only four transmission cases are considered hereafter. Let $\mu$ be the average throughput from the wired backhaul to each UE. Hence, we have

$$
\mu=P_{d} \mu_{d}+P_{r} \mu_{r}+P_{r d} \mu_{r d}+P_{r r} \mu_{r r},
$$

where $P_{d}, P_{r}, P_{r d}$, and $P_{r r}$ are the probabilities for transmission cases 1 to 4 , and $\mu_{d}, \mu_{r}, \mu_{r d}$, and $\mu_{r r}$ are the average throughputs of transmission cases 1 to 4 , respectively.

Besides transmission case 1, the average throughput for transmission cases 2 to 4 can be evaluated by the minimum value of average transmission rate among all links. Let $E\left\{D_{0, j}\right\}$ be the average transmission rate of the access link from DeNB to UE $j, E\left\{A_{\tau(j), j}\right\}$ be the average transmission rate of the relay link from DeNB to target $R N_{\tau(j)}$ and $E\left\{D_{\tau(j), j}\right\}$ be the average transmission rate of the access link from the target $R N_{\tau(j)}$ to UE $j$. Hence, we have

$$
\begin{gathered}
\mu_{d}=\frac{1}{M} \sum_{j=1}^{M} \alpha_{A} E\left\{D_{0, j}\right\}, \\
\mu_{r}=\frac{1}{M} \sum_{j=1}^{M} \min \left(\alpha_{R} E\left\{A_{\tau(j), j}\right\}, \alpha_{A} E\left\{D_{\tau(j), j}\right\}\right), \\
\mu_{r d}=\frac{1}{M} \sum_{j=1}^{M} \min \left(\rho_{R} \eta_{f} E\left\{U_{\sigma(j), j}\right\}, \alpha_{A} E\left\{D_{0, j}\right\}\right), \\
\mu_{r r}=\frac{1}{M} \sum_{j=1}^{M} \min \left(\rho_{R} \eta_{f} E\left\{U_{\sigma(j), j}\right\}, \alpha_{R} E\left\{A_{\tau(j), j}\right\},\right. \\
\left.\alpha_{A} E\left\{D_{\tau(j), j}\right\}\right),
\end{gathered}
$$

where $\alpha_{R}, \alpha_{A}$ are the portion of frame reserved by the DeNB for the downlink transmission of the relay link, the downlink transmission of the access link and $\rho_{R}$ is the uplink transmission of the relay link. The values of $\alpha_{R}, \alpha_{A}$, and $\rho_{R}$ depend on the time division duplex (TDD) subframe configurations [11] and can be set as in [4]. The term $E\left\{U_{\sigma(j), j}\right\}$ denotes the average uplink transmission rate of the relay link from serving RN $\sigma(j)$ to the DeNB. The expected value can be obtained by the time average value, i.e., $E\left\{U_{\sigma(j), j}\right\}=$ $\lim _{T \rightarrow \infty} \frac{1}{T} \sum_{k=1}^{T} U_{\sigma(j), j}[k]$. The term $\eta_{f}$ denotes the portion of uplink bandwidth reserved for the forwarded packets during handover, where $0 \leq \eta_{f} \leq 1$. It is worth noting, however, that $\eta_{f}$ cannot be set to 1 because part of the uplink bandwidth should be allocated for the transmission of UE's uplink data, not always for the transmission of forwarded packets during the handover. Hence, the value of $\eta_{f}$ is usually smaller than 1 .

Note that $P_{d}$ and $P_{r}$ depend only on the pre-defined handover thresholds. However, $P_{r d}$ and $P_{r r}$ depend not only on the pre-defined handover thresholds but also on the number of forwarded packets buffered at the old serving RN. $P_{d}$ and $P_{r}$ are the special cases of $P_{r d}$ and $P_{r r}$, respectively, when the length of the pre-buffering queue at the old serving $\mathrm{RN}$ is not equal to zero. Hence, we have

$$
\begin{array}{r}
P_{d}=\operatorname{Pr}\left\{\gamma_{0, j}-\gamma_{\tau(j), j}>\gamma_{H O} \mid \tau(j) \neq 0\right\}, \\
P_{r}=\operatorname{Pr}\left\{\gamma_{\sigma(j), j}-\gamma_{\tau(j), j}>\gamma_{H O} \mid \sigma(j) \neq 0,\right. \\
\tau(j) \neq \sigma(j)\}, \\
P_{r d}=\operatorname{Pr}\left\{\gamma_{0, j}-\gamma_{\tau(j), j}>\gamma_{H O}, B_{\sigma(j), j}[k] \neq 0 \mid\right. \\
\left.\tau(j) \neq 0, \sigma(j) \neq 0, k \in H_{j}\right\} \\
=P_{d} \times \operatorname{Pr}\left\{B_{\sigma(j), j}[k] \neq 0 \mid \sigma(j) \neq 0, k \in H_{j}\right\}, \\
P_{r r}=\operatorname{Pr}\left\{\gamma_{\sigma(j), j}-\gamma_{\tau(j), j}>\gamma_{H O}, B_{\sigma(j), j}[k] \neq 0 \mid\right. \\
\left.\sigma(j) \neq 0, \tau(j) \neq \sigma(j), k \in H_{j}\right\} \\
=\operatorname{Pr} \times \operatorname{Pr}\left\{B_{\sigma(j), j}[k] \neq 0 \mid \sigma(j) \neq 0, k \in H_{j}\right\},
\end{array}
$$

where $\gamma_{H O}$ and $\operatorname{Pr}\left\{B_{\sigma(j), j}[k] \neq 0 \mid \sigma(j) \neq 0, k \in H_{j}\right\}$ are the handover threshold (in $\mathrm{dB}$ ) and the probability that the serving RN has packets to be forwarded to DeNB after performing the handover, respectively. Note that $\operatorname{Pr}\left\{B_{\sigma(j), j}[k] \neq\right.$ $\left.0 \mid \sigma(j) \neq 0, k \in H_{j}\right\}$ increases as the average number of forwarded packets rises.

\section{Pre-buffering-AWARe Flow Control Scheme (PFCS)}

This section explains the PFCS. There are two phases in the proposed scheme. The first phase is the flow control phase. Here, the DeNB proceeds to execute the dynamic flow control algorithm [4] as its flow control functionality to avoid buffer overflow and buffer underflow problems at RNs. The other phase is the pre-buffering phase, where DeNB starts to trigger the pre-buffering functionality to multicast data packets to the candidate $\mathrm{RN}(\mathrm{s})$ when the threshold of pre-buffering functionality is reached.

Figure 2 and 3 shows the message sequence charts of the flow control phase and the pre-buffering phase, respectively. In the flow control phase of the proposed scheme (as shown in Fig. 2), the RN can dynamically feed back the report message (with a window size $W_{i, j}[k]$ ) to notify DeNB to control the arrival rate of the serving $\mathrm{RN}$. In the other phase, as shown in Fig. 3, the DeNB can decide whether to trigger the pre-buffering functionality to multicast its data packets to the candidate $\mathrm{RN}(\mathrm{s})$, which involves the target $\mathrm{RN}$. If the relative signal quality between the serving and the target 


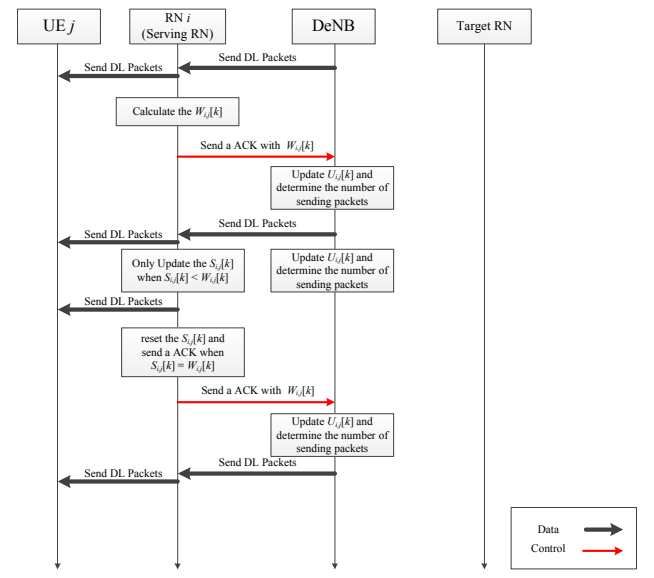

Fig. 2. Message sequence chart of flow control phase for the proposed scheme.

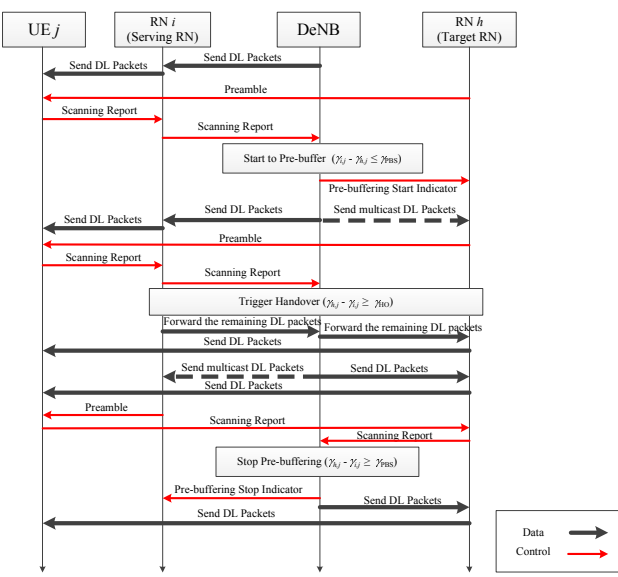

Fig. 3. Message sequence chart of pre-buffering phase for the proposed scheme.

RNs is less than or equal to the pre-buffering threshold (i.e., $\left.\gamma_{i, j}-\gamma_{h, j} \leq \gamma_{P B S}\right)$, the DeNB will send an indicator to trigger the pre-buffering functionality and multicast the UE's data packets from the DeNB to the candidate $\mathrm{RN}(\mathrm{s})$ and the serving RN simultaneously. When the UE is very close to the target $\mathrm{RN}$ and is far away from the serving $\mathrm{RN}$ (i.e., $\left.\gamma_{h, j}-\gamma_{i, j} \geq \gamma_{P B S}\right)$, the DeNB sends another indicator to cancel the multicast transmission.

\section{Simulation Results}

Simulations were conducted on top of a C-based platform to verify the effectiveness of the proposed scheme. In the simulations, each point represented the mean value of 1000 samples. Based on [1], we deployed a DeNB with coverage of 866 meters. Three RNs were located at the edge of each DeNB. Twenty mobile UEs were randomly distributed in the coverage of the DeNB and followed the Random Way Point (RWP) mobility model with a speed of $60 \mathrm{~km} / \mathrm{hr}$. A DeNB and an $\mathrm{RN}$ have maximum transmission power of $46 \mathrm{dBm}$ and $30 \mathrm{dBm}$, respectively. The path-loss models were based on 3GPP TR 36.814 [1]. The subframe configuration number 4 of frame structure type 2 (i.e., TDD) [11] was considered

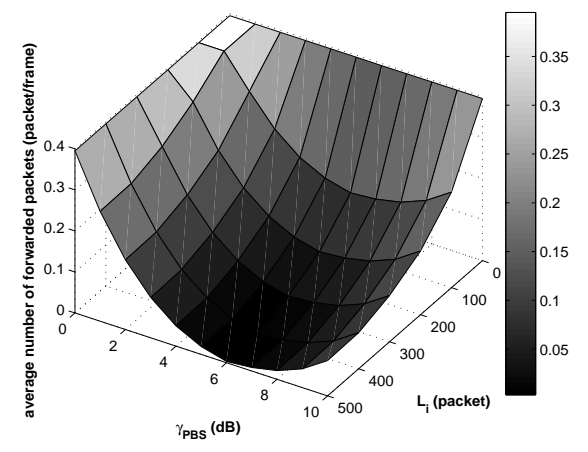

Fig. 4. Average number of forwarded packets for the proposed scheme with different combination of the pre-buffering length and the pre-buffering threshold.

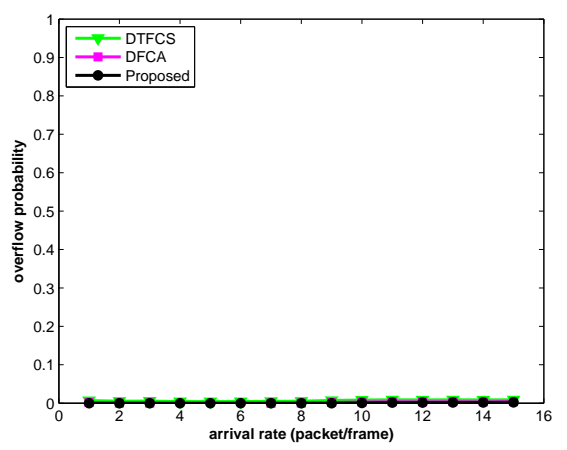

Fig. 5. Buffer overflow probability for three schemes.

for the following simulations. That is, the setting of $\alpha_{R}, \alpha_{A}$, and $\rho_{R}$ are $0.2,0.2$, and 0.2 , respectively.

In this paper, we focus on the performance evaluation in the downlink transmission. The transmission rate of the relay link for each UE was controlled by the specified flow control scheme. The transmission rate of access link for each UE followed the AMC scheme and was based on the UE's signal quality (i.e., $\gamma_{i, j}$ ). Table I shows the parameters of the MCS when the $B E R=10^{-6}$. The handover threshold $\gamma_{H O}$ and pre-buffering threshold $\gamma_{P B S}$ are $0 \mathrm{~dB}$ and $6 \mathrm{~dB}$, respectively. The portion of uplink bandwidth allocated to the forwarded packets is 0.5 (i.e., $\eta_{f}=0.5$ ). The total length of the local queue reserved for each UE is 50 packets (i.e., $K_{i, j}=50$ ).

In the following simulations, the size of pre-buffering queue $L_{i}=400$ is considered. DFCA in [4] and the DTFCS in [5] are chosen as benchmarks to compare with our proposed scheme. Figure 5 to 8 shows the comparison performance of DFCA, the DTFCS and the proposed scheme for buffer overflow probability, buffer underflow probability, average number of forwarded packets and average throughput, respectively. In these figures, we still observe the arrival rate of UE $\lambda_{j}$ ranging from 1 to 15 packets/frame.

Figure 5 and 6 shows the buffer overflow and buffer underflow probabilities of the local queue, respectively. In Fig. 5, we find that these three schemes can well prevent buffer overflow 


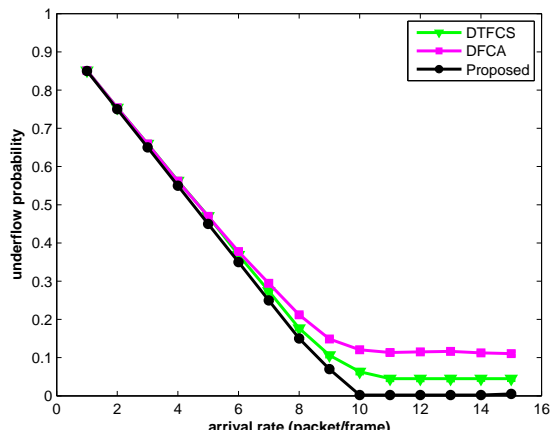

Fig. 6. Buffer underflow probability for three schemes.

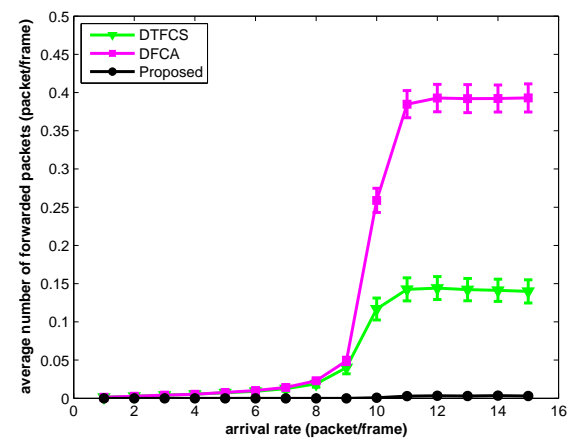

Fig. 7. Average number of forwarded packets for three schemes.

in the local queue, as the buffer overflow probabilities of the three schemes are zero. In Fig. 6, the results show that the buffer underflow probabilities of these schemes for $\lambda_{j} \leq 10$ decrease when the UE's arrival rate increases. Additionally, we find that the DTFCS and the DFCA have higher buffer underflow probabilities. This is because these two schemes cannot completely solve the handover forwarding problems by reducing the forwarding traffic and have lower forwarding rates to deliver the forwarded packets. On the other hand, the proposed scheme can minimize the average number of forwarded packets so that it has optimal performance in terms of the buffer underflow probability.

Figure 7 and 8 shows the average number of forwarded packets and the average throughput, respectively. Both figures show that the DTFCS has better average throughput than the DFCA because the DTFCS can reduce the average number of forwarded packets before the handover. In addition, the results show that the proposed scheme has the best performance in terms of average throughput, because almost zero packets were forwarded from the serving $\mathrm{RN}$, whereas both the DFCA and the DTFCS had a higher average number of forwarded packets when the network was congested (i.e., arrival rate $\lambda_{j} \geq 10$ packets/frame).

\section{Conclusions}

In this paper, we propose a PFCS to solve the buffer overflow, buffer underflow, and handover forwarding problems

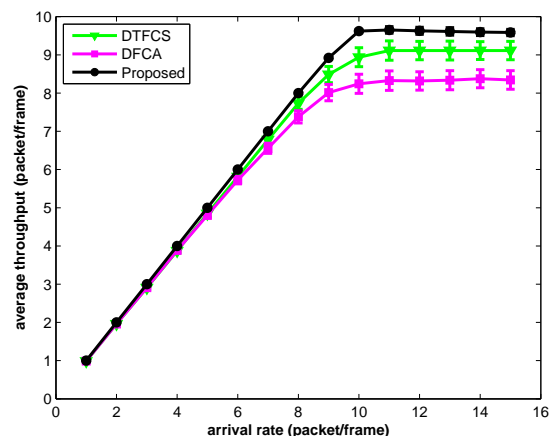

Fig. 8. Average throughput for three schemes.

and show its effectiveness in an LTE-A relay network. The results indicate that the proposed scheme can prevent the buffer overflow and the buffer underflow of the local queue at RNs and throughput degradation due to the large number of forwarded packets. We first provide the optimal configuration for the proposed scheme to ensure the best average throughput under the condition of minimal implementation cost. Eventually, we found that the proposed scheme can outperform the traditional flow control schemes because the average number of forwarded packets is minimized.

\section{ACKNOWLEDGEMENT}

This work was supported in part by the Ministry of Science and Technology, Taiwan, R.O.C., under Contract MOST 1022221-E-011-003-MY3 and MOST 103-2221-E-182-042, and by the "Aiming For the Top University Program" funded by Ministry of Education, Taiwan, R.O.C.

\section{REFERENCES}

[1] 3GPP TR 36.814, Evolved universal terrestrial radio access (E-UTRA): Further advancements for E-UTRA physical layer aspects, June 2009.

[2] P. Bhat et al., LTE-Advanced: An operator perspective, IEEE Communications Magazines, 50 (2) (2012) pp. 104-114.

[3] Z. Ma, W. Zian, H. Long, and W. Wang, Proportional fair resource partition for LTE-Advanced networks with type I relay nodes, in: Proceedings of IEEE ICC, Kyoto, Japan, June 2011.

[4] P. C. Lin, R. G. Cheng, and Y. J. Cheng, A dynamic flow control algorithm for LTE-Advanced relay networks, IEEE Transactions on Vehicle Technology, 63 (1) (2014) pp. 334-343.

[5] P. C. Lin and R. G. Cheng, Dynamic two-threshold flow control scheme for 3GPP LTE-A relay networks, in: Proceeding of IEEE PIMRC, London, UK, Sep. 2013, pp. 2664-2668.

[6] N. Badache and D. Tandjaoui, A seamless handoff protocol for hierarchical mobile IPv4, in: Proceeding of 4th International Workshop Mobile and Wireless Communications Network, Sep. 2002, pp. 651-655.

[7] M. Tanno, A. Morimoto, K. Higuchi, and M. Sawahashi, Experiments on handover using combination of bicast and forwarding of IP packet for future IP-based RANs, in: Proceeding of IEEE PIMRC, Athens, Greece, Sep. 2007, pp. 1-5.

[8] P. Lin, Y. B. Lin, and I. Chlamtac, Overflow control for UMTS high-speed downlink packet access, IEEE Transactions on Wireless Communications, 3 (2) (2004), pp. 524-532.

[9] J. Y. Kim and D. H. Cho, Pre-buffering scheme for seamless relay handover in relay based cellular system, in: Proceeding of IEEE VTCSpring, Barcelona, Spain, April 2009, pp. 1-5.

[10] B. Can et al., Implementation issues for OFDM-Based multihop cellular networks, IEEE Communications Magazines, 45 (9) (2007), pp. 74-81.

[11] 3GPP TS 36.216, Evolved universal terrestrial radio access (E-UTRA): Physical layer for relaying operation, Mar. 2011. 